\title{
MicroRNA-218 Increases the Sensitivity of Bladder Cancer to Cisplatin by Targeting Glut1
}

\author{
Peng Lia Xiao Yang ${ }^{a} \quad$ Yidong Cheng $^{\mathrm{b}} \quad$ Xiaolei Zhang ${ }^{\mathrm{a}}$ Chengdi Yang ${ }^{\mathrm{a}}$ \\ Xiaheng Deng ${ }^{a}$ Pengchao $\mathrm{Li}^{\mathrm{a}}$ Jun Tao ${ }^{\mathrm{a}}$ Haiwei Yang ${ }^{\mathrm{a}}$ Jifu Weic Jingyuan Tang ${ }^{\mathrm{a}}$ \\ Wenbo Yuan ${ }^{a}$ Xiaoting Xua Qiang Lu ${ }^{a}$ Min Gu \\ aDepartment of Urology, The First Affiliated Hospital of Nanjing Medical University, Nanjing, \\ 'Department of Urology, The Third Affiliated Hospital of Nanjing University of Chinese Medcine, \\ Nanjing, 'Research Division of Clinical Pharmacology, The First Affiliated Hospital of Nanjing Medical \\ University, Nanjing, China
}

\section{Key Words}

MiR-218 • Glut1 • Cisplatin • Chemo-sensitivity • Bladder cancer

\begin{abstract}
Background/Aims: MicroRNA-218 (miR-218) is down-regulated in many malignancies that have been implicated in the regulation of diverse processes in cancer cells. However, the involvement of miR-218 in chemo-sensitivity to cisplatin and the precise mechanism of this action remained unknown in bladder cancer. Methods: qRT-PCR was used to detect miR-218 and its target Glut1 expression in bladder cancer cell lines T24 and EJ. CCK-8 method was utilized to measure the cell viability. IC 50 was calculated via a probit regression model. Glut1 was detected by western blotting for analysis of potential mechanism. Luciferase reporter assay was utilized to validate Glut1 as a direct target gene of miR-218. The intracellular level of GSH and ROS were determined using a commercial colorimetric assay kit and 2', 7'-dichlorodihydro-fluorescein diacetate, respectively. Results: Over-expression of miR-218 significantly reduced the rate of glucose uptake and total level of GSH and enhanced the chemo-sensitivity of bladder cancer to cisplatin. Mechanistically, Glut1 was found to be a direct and functional target of miR-218. Up-regulation of Glut1 could restore chemo-resistance in T24 and EJ cells. On the contrary, knockdown of Glut1 could generate a similar effect as up-regulating the expression of miR-218. Conclusions: MiR-218 increases the sensitivity of bladder cancer to cisplatin by targeting Glut1. Restoration of miR-218 and repression of glut1 may provide a potential strategy to restore chemo-sensitivity in bladder cancer.

\section{Introduction}

Bladder cancer is a common disease worldwide; approximately 356,000 new cases are diagnosed each year and roughly 145,000 deaths from the disease are reported annually [1].

P. Li, X. Yang and Y. Cheng contributed equally to this work.

Qiang Lu and Min Gu Department of Urology, The First Affiliated Hospital of Nanjing Medical University, 


\section{Cellular Physiology Cell Physiol Biochem 2017;41:921-932 \begin{tabular}{ll|l} 
DOI: 10.1159/000460505 & O 2017 The Author(s). Published by S. Karger AG, Basel \\
and Biochemistry & Publisned on ine:-February 20, 2017 www.karger.com/cpb
\end{tabular} \\ Li et al.: Sensitization of Bladder Cancer to Cisplatin by miR-218-Glut1 Pathway}

What's more, $10 \%-20 \%$ of all recurring patients are at a high risk of progressing to muscleinvasive bladder cancer (MIBC) [2]. Chemotherapy is one of the most important treatments utilised in bladder cancer to reduce relapse, prevent progress and improve survival. Although cisplatin-containing combination chemotherapy has been the standard of care since the late 1980s [3], the cisplatin/gemcitabine (GC) regimen has a median time-to-progression of only 6 months and has no effect on overall survival after radical cystectomy in high-risk patients [4]. In addition, chemotherapy has failed in a large proportion of patients because of chemotherapy resistance, which leads to the relapse and progression of tumours. Therefore, chemotherapy resistance has become a major obstacle to the success of cancer management.

cis-Diaminedichloroplatinum (II), also known as cisplatin, is a platinum-based drug employed to manage a wide range of malignancies, including bladder cancer [5]. Cisplatin mediates anticancer effects via multiple mechanisms, the most prominent of which is inducing oxidative stress [6]. Nevertheless, many cancers demonstrate chemo-resistance towards cisplatin treatment. Because chemo-resistance, numerous of cisplatin-treated patients must tolerate various side effects without actually benefiting from chemotherapy. Various of mechanisms, such as reduced uptake, increased efflux, increased inactivation of ROS and increased levels of intracellular GSH, amongst others [7] are involved in this phenomenon. Recent studies indicate that microRNAs (miRNAs) may regulate these processes [8].

MiRNAs are endogenous, short non-coding RNAs of approximately 18-25 nt in length. MiRNAs negatively regulate protein-coding gene expression post-transcriptionally by targeting the mRNA 3'-untranslated regions (3'-UTR) [9, 10]. The association between miRNAs and tumour chemo-sensitivity has recently attracted increased attention [8]. Some research has shown that miR-218 acts as a tumour-suppressing miRNA in many cancers, including glioma, cervical cancer and colon cancer [11-13]. Our previous study showed that miR-218 expression was down-regulated in clinical bladder cancer tissues and inhibited the proliferation, migration and invasion of bladder cancer cells [14]. However, the association between miR-218 and the sensitivity of bladder cancer to cisplatin-based treatment remains unrevealed.

Glut1(Glucose transporter isoform 1) is a key rate-limiting enzyme in controlling glycolytic flux in cells [15] and plays a crucial role in tumourigenesis and progression [16]. Glut1 is over-expressed and associated with poor prognosis in varieties of malignancies including bladder cancer [17-19]. Over-expression of Glut1 enhances glycolytic activity, increases cancer cell proliferation and promotes the invasion and metastatic proficiency of cancers $[20,21]$. However, the role of Glut1 in the development of cisplatin resistance in bladder cancer remains unknown.

In this study, we investigate the relationship between miR-218 and the sensitivity of bladder cancer cells to cisplatin. Glut1 is also predicted and confirmed as a target gene of miR-218. Our study contributes to the further understanding of the role of miR-218 in bladder cancer and provides a novel option for treatment of bladder cancer.

\section{Materials and Methods}

\section{Cell culture}

The human bladder cancer cell lines T24 and EJ were purchased from the Type Culture Collection of the Chinese Academy of Sciences (Shanghai, China) and maintained in DMEM medium containing 10\% foetal bovine serum (FBS; Gibco, Australia) and 1\% penicillin-streptomycin. All cells were cultured in an incubatorwith humidified $5 \% \mathrm{CO}_{2}$ at $37^{\circ} \mathrm{C}$.

Transfection

All of the miRNA mimics and siRNA used for transfection were synthesised by GenePharma (Shanghai, China). The Glut1 overexpression and non-sense plasmids were constructed by GeneCopoeia(Shanghai, China). Cells at about 70\% confluence were seeded into six-well plates and transfected with $50 \mathrm{nM}$ mature miR-218 mimics, Glut1 over-expression plasmid or Glut1 siRNA and their non-sense controls 


\section{Cellular Physiology Cell Physiol Biochem 2017;41:921-932 \begin{tabular}{c|c|c|c|} 
DOI: 10.1159/000460505 & O 2017 The Author(s). Published by S. Karger AG, Basel \\
and Biocherger.com/cpb
\end{tabular} \\ Li et al.: Sensitization of Bladder Cancer to Cisplatin by miR-218-Glut1 Pathway}

using Lipofectamine 2000 or Lipofectamine 3000 transfection reagent (Invitrogen, USA) according to the manufacturer's protocol. The cells were harvested $48 \mathrm{~h}$ after transfection.

\section{Plasmid constructs and luciferase reporter assay}

To construct the Glut1 expression plasmids, the wide-type or mutant 3'UTR of Glut1 gene was cloned into the pEZX vector. For the luciferase reporter assay, T24 cells were seeded into 24 -well plates and incubated overnight. The T24 cells were then co-transfected with plasmid containing pEZX /Glut1-3'-UTR or pEZX/Glut1-3'-UTR-mutant and miR-218 mimics or the control using Lipofectamine 2000 (Invitrogen). After $24 \mathrm{~h}$ of transfection, the cells were lysed and Firefly and Renilla luciferase activities were measured by a microplate reader using Luc-Pair miR Luciferase Assay (GeneCopoeia, FulenGen, China). Normalised data were obtained by calculating the ratios of Firefly to Renilla luciferase luminescence. All experiments were performed in triplicate.

$I C_{50}$ determination

Twenty-four hours after transfection, cells were digested and seeded into 96-well plates at a density of 5000 cells per well in triplicate and incubated overnight. Then, the cells were treated with a series of dilute concentrations of cisplatin (Sigma). Cell viability was measured after $48 \mathrm{~h}$ using the CCK-8 method (CCK8, Dojindo, Japan) according to the manufacturer's instructions. The probit regression model was utilised to calculate the $\mathrm{IC}_{50}$ as previous described [22]. All experiments were independently performed three times.

Measurement of intracellular reactive oxygen species

The intracellular reactive oxygen species (ROS) were measured using the fluorogenic reagent 2', 7'-dichlorodihydro-fluorescein diacetate (Invitrogen) according to the manufacturer's protocols. DCFHDA substrate presents no fluorescence by itself and is deacetylated into dichlorofluorescin (DCFH) intracellularly by esterase. DCFH is further oxidised into the fluorescent compound dichlorofluorescein (DCF) by intracellular peroxides. Cells were seeded into 96-well plates at a density of 5000 cells per well in triplicate and incubated overnight. The cells were washed twice with PBS and incubated in $10 \mu \mathrm{M}$ DCFH-DA at $37^{\circ} \mathrm{C}$ for $30 \mathrm{~min}$. The fluorescence intensity of the incubation solution was measured at $530 \mathrm{~nm}$ using a microplate reader.

Determination of total cellular glutathione content

Intracellular levels of reduced glutathione (GSH) were determined using a GSH colorimetric assay kit (BioVision, USA) according to the manufacturer's instructions. Briefly, $48 \mathrm{~h}$ after transfection, cells were harvested, washed twice with PBS, disrupted using ultrasound and centrifuged at $800 \times \mathrm{g}$ for $10 \mathrm{~min}$ at $4^{\circ} \mathrm{C}$. Supernatants were isolated and detected for total intracellular GSH by mixing with dithiobisnitrobenzoic acid (DTNB). Absorbance was measured at $405 \mathrm{~nm}$ using a microplate reader.

\section{RNA extraction and $q R T-P C R$}

Total RNA and miRNA were extracted from transfected cells using Trizol reagent (Invitrogen) and a miRNeasy Mini Kit (Qiagen), respectively. Real-time qRT-PCR was performed to detect the expressions of Glut1 and miR-218 by using SYBR Green PCR Kit (Takara, Japan) according to the manufacturer's protocol. Briefly, 500 ng total RNA was used for the initial reverse transcription reaction with the gene-specific stemloop RT primer available in the kit. Here, $\beta$-actin and U6 RNAs were used as internal controls for mRNA and miRNA detection, respectively. The qPCR primers were obtained from Sango Biotech (Shanghai, China), and the sequences were as follows: Glut1 (forward: 5'-AAC TCT TCA GCC AGG GTC CAC-3'; reverse: 5'-CAC AGT GAA GAT GAA GAC-3'); $\beta$-actin (forward 5'-AGC GAG CAT CCC CCA AAG TT-3'; reverse 5'-GGG CAC GAA GGC TCA TCA TT-3'). Each experiment was replicated three times and data were analysed using the $2^{-\triangle \Delta C T}$ method.

\section{Protein extraction and Western blot analysis}

The primary antibodies used in this study, including Glut1 and $\beta$-actin, were products of Cell Signalling Technology (CST, USA). The transfected cells were lysed and total protein was extracted using RIPA lysis buffer (Beyotime, China). Protein concentration was quantified and a total of $40 \mu \mathrm{g}$ of proteins was loaded onto $10 \%$ SDS-PAGE gels. After electrophoresis, the proteins were transferred to PVDF membranes. The 


\section{Cellular Physiology Cell Physiol Biochem 2017;41:921-932

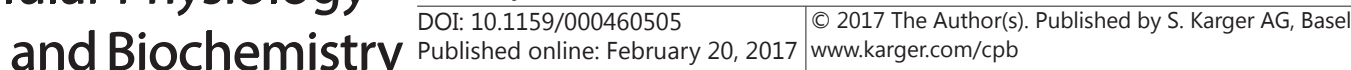 \\ Li et al.: Sensitization of Bladder Cancer to Cisplatin by miR-218-Glut1 Pathway}

membranes were blocked for $2 \mathrm{~h}$ at room temperature with 5\% non-fat milk in TBST and then incubated with primary antibodies at $4^{\circ} \mathrm{C}$ overnight. The membranes were then washed three times in TBST and incubated with horseradish peroxidase-conjugated secondary antibody at room temperature for $1 \mathrm{~h}$. The blots were developed with ECL solution (Pierce, Rockford, IL, USA) and detected by using a chemiluminescence system (Bio-Rad, USA). Image Lab Software was employed to analyse the intensities of the band signals obtained.

Glucose uptake assay

Approximately $24 \mathrm{~h}$ before the glucose uptake experiments, cells were plated on six-well plates at a density of $3 \times 10^{5}$ cells/well, washed twice with phosphate-buffered saline (PBS), and then incubated in 2 $\mathrm{ml}$ of Krebs-Ringer-HEPES (KRH) buffer (25 mM Hepes, pH 7.4, $120 \mathrm{mM} \mathrm{NaCl}, 5 \mathrm{mM} \mathrm{KCl}, 1.2 \mathrm{mM} \mathrm{MgSO}{ }_{4}, 1.3$ $\mathrm{mM} \mathrm{CaCl}_{2} 1.3 \mathrm{mM} \mathrm{KH}_{2} \mathrm{PO}_{4}$ ) containing $1 \mu \mathrm{Ci}$ of [3H]-2-deoxyglucose (Perkin Elmer Life Sciences) at $37^{\circ} \mathrm{C}$ for $20 \mathrm{~min}$ to initiate the experiments. The cells were consequently washed twice with ice-cold KRH buffer to halt uptake and lysed in $300 \mu \mathrm{l}$ of lysis buffer [10 mM Tris-HCl, pH 8.0, 0.2\% sodium dodecyl sulphate (SDS)]. Radioactivity was measured by liquid scintillation spectrometry. Disintegrations per minute (DPM) were used to evaluate the intracellular level of [3H]-2-deoxyglucose, and each assay was performed in triplicate.

\section{Statistical analysis}

All data are presented as mean \pm standard deviation. Statistical analysis was performed using SPSS software (version 17.0; IBM Corporation). Student's $t$ test was utilised to analyse differences between two groups. Statistical significance was considered at 0.05. Graphical presentations were performed using GraphPad Prism 5 software (San Diego, CA). All experiments were repeated more than three times, and each experiment was performed in triplicate.

\section{Results}

Over-expression of microRNA-218 restores drug sensitivity

To investigate the effects of miR-218 on bladder cancer cell lines, we transfected miR218 mimics or the non-sense control (NC) into T24 and EJ cells. RT-PCR was performed to confirm that miR-218 was significantly increased in the miR-218 mimics group compared with that in the NC group (Fig. 1A). CCK-8 assay showed that compared with NC, overexpression of miR-218 could dramatically inhibit the proliferation of T24 and EJ cells in a series of dilute concentrations of cisplatin (Fig. 1B). These results indicate that increased expression of miR-218 enhanced cisplatin sensitivity in T24 and EJ cells (Fig. 1C).

Altered metabolism (the Warburg effect), which manifests as increased glucose uptake and decreased oxidative phosphorylation, is considered a hallmark of cancer. We determined whether overexpression of miR-218 could affect the metabolism of T24 and EJ cells. Glucose uptake assay suggested that miR-218 over-expression significantly decreased the rate of glucose uptake (Fig. 1D) compared with the control. Reduced glutathione (GSH) is a major cellular metabolite that protects against oxidative and chemical injury and exhibits a variety of other cytoprotective effects. High levels of GSH as well as increased expression of antioxidant enzymes promote cancer cell survival and resistance to anticancer agents [23]. We detected the intracllular level of GSH by utilising a reduced GSH assay and found that over-expressed miR-218 inhibited the generation of intracellular GSH (Fig. 1E). Intracellular ROS levels were also measured and results implied that miR-218 increased the production of ROS (Fig. 1F). All these results imply that miR-218 could promote sensitivity to cisplatin in the T24 and EJ cell lines via various mechanisms.

MicroRNA-218 directly targets Glut1 3'-UTR and negatively regulates its expression

Glut1, a key rate-limiting enzyme in the transport of glucose into cancer cells, is overexpressed in many cancers. Our previous study found that Glut1 was dramatically overexpressed in clinical bladder cancer tissue whereas miR-218 was markedly down-regulated $[14,24]$. Therefore, we sought to determine whether Glut1 is regulated by miR-218. qRT-PCR and Western blot assay were performed to detect the effect of miR-218 on the expression 
A

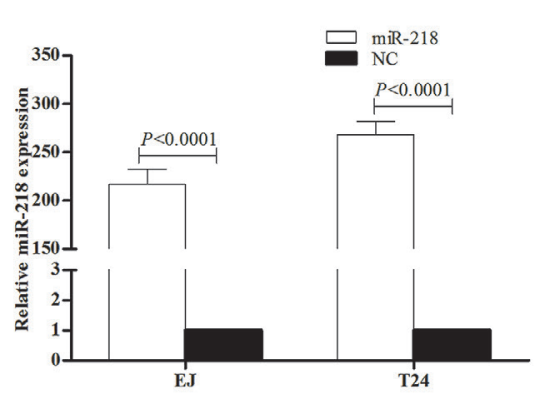

C

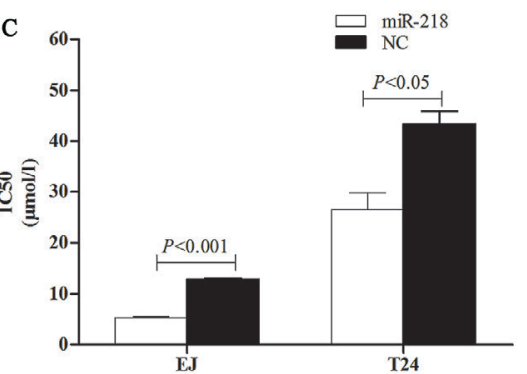

$\mathrm{E}$

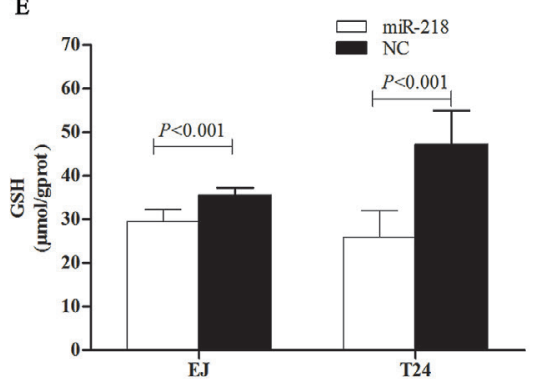

B
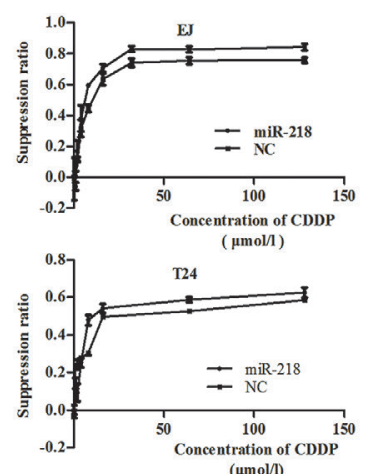

D

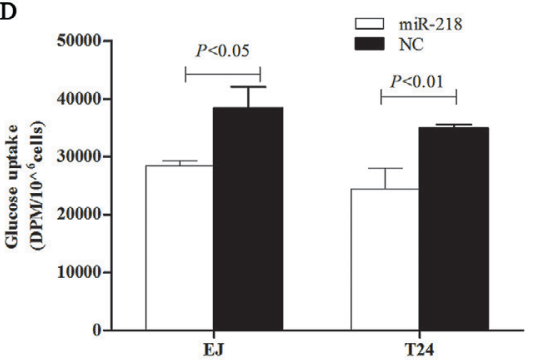

$\mathbf{F}$

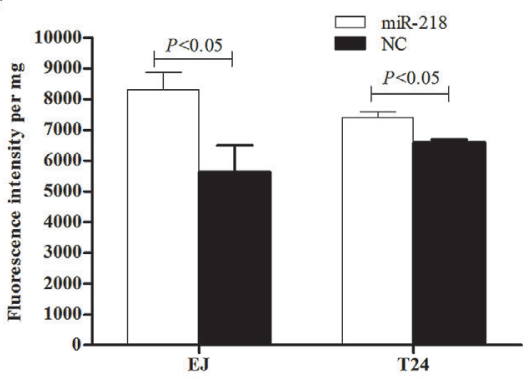

Fig. 1. Over-expression of microRNA-218 restores drug sensitivity in T24 and EJ cells. (A) miR-218 expression levels in miR-218-transfected and miR control-transfected cells. (B) Cells viability was determined by the CCK-8 method to be inhibited by miR-218-transfected cells in a series of dilute concentrations of cisplatin. (C) Transfection of miR-218 increased the sensitivity of cells to cisplatin. (D) Overexpression of miR-218 decreased the rate of glucose uptake. (E, F) Reduced GSH and increased levels of ROS were observed in miR-218-transfected cells.

of Glut1, and results demonstrated that miR-218 induced significant decreases in Glut1 compared with the NC group at both the mRNA and protein levels (Fig. 2B, 2D).

Utilising the programs picTar, TargetScan (http://www.targetscan.org) and miRNA (http:// www.microrna.org), potential binding sites in the 3'UTR of Glut1 were found (Fig. 2A). To investigate whether the Glut1 3'-UTR is a direct target of miR-218 further, we constructed vectors encoding the full length of the 3'-UTR of Glut1 mRNA to perform a dual luciferase reporter assay (Fig. 2C). Co-transfection experiments implied that miR-218 significantly reduced the luciferase activity of Glut1 containing a wild-type 3'-UTR but not that of Glut1 containing mutant-type 3'-UTR (Fig. 2C). These results confirmed that Glut1 is negatively regulated by miR-218 and is a direct target of miR-218.

Knockdown of Glut1 increases the sensitivity to cisplatin in T24 and EJ cells

Our work showed in Fig. 2 confirmed that miR-218 depresses the expression of Glut1 at both the mRNA and protein levels by binding to its 3'UTR. However, the role of Glut1 in the development of cisplatin resistance remains unknown. We performed loss-of-function and gain-of-function experiments to study the Glut1 gene more extensively. T24 and EJ cells 
A

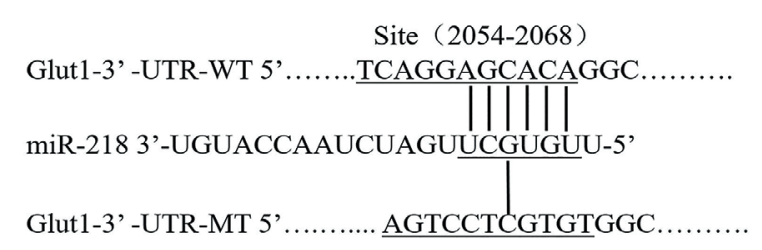

B

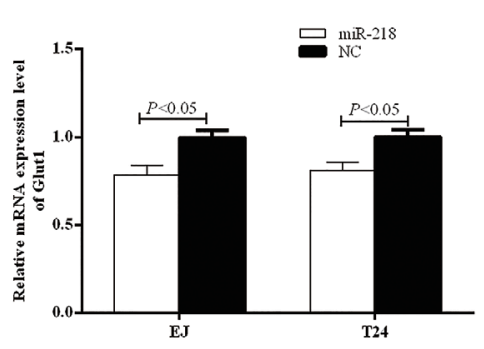

D

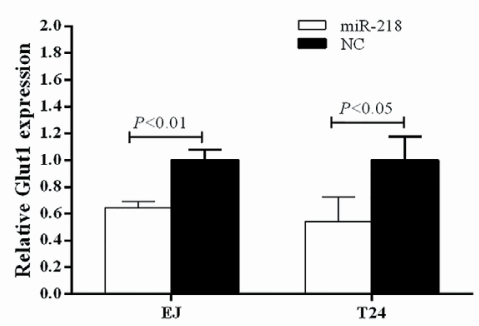

C
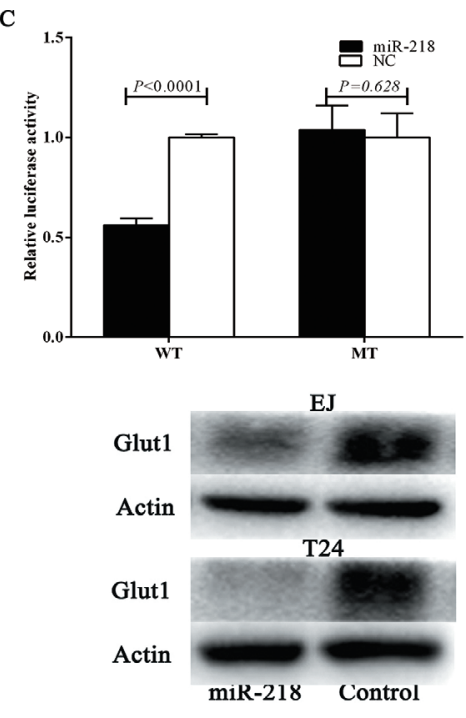

Fig. 2. MicroRNA-218 directly targets the Glut1 $3^{\prime}$-UTR and negatively regulates its expression. (A) Putative miR-218-binding sequence of the $3^{\prime}$-UTR of Glut1 mRNA. (B) mRNA levels of Glut1 in miR-218-transfected and miR control-transfected cells. (C) Dual luciferase reporter assays demonstrated that miR-218 over-expression could reduce the fluorescence intensity in T24 cells co-transfected with miR-218 mimics or the control and the wild-type Glut1 3'-UTR vector (WT); similar effects were not observed with the mutant type Glut1 3'-UTR vector (MT). (D) Over-expression of miR-218 significantly reduced the expression of Glut1 protein.

were transfected with small-interfering (si) Glut1 or NC, and the mRNA and protein levels of Glut1 were subsequently detected by qRT-PCR and Western blot. The results showed the depressed expression of Glut1 (Fig. 3A, 3B). Compared with the NC group, cells transfected with si-Glut1 showed significantly inhibited viability and more sensitivity to cisplatin in a series of dilute concentrations (Fig. 3C, 3D). As well, the results of reduced GSH assay suggested that intracellular GSH contents dramatically decreased by knockdown of Glut1 in T24 and EJ cells (Fig. 3E). Blockage of Glut1 also markedly promoted ROS generation (Fig. $3 \mathrm{~F}$ ). These results were consistent in cells over-expressing miR-218 and suggested that miR218 regulates cisplatin chemo-sensitivity in bladder cancer by targeting Glut1.

Over-expressed Glut1 regains drug-resistance in T24 and EJ cells

To study the function of Glut1 in regulating the chemo-sensitivity of bladder cancer cells to cisplatin, we examined the effect of over-expressed Glut1 in T24 and EJ cells. qRT-PCR and Western blot analysis verified that the expression of Glut1 was significantly enhanced at both the mRNA and protein levels (Fig. 4A, 4B). In addition, up-regulated expression of Glut1 promoted the proliferation of T24 and EJ cells and restored their chemo-resistance to cisplatin (Fig. 4C, 4D). Moreover, increased generation of intracellular GSH and decreased levels of ROS were observed in cells transfected with Glut1 plasmids (Fig. 4E, 4F). All of these results indicated that miR-218 enhances the chemo-sensitivity of bladder cancer to cisplatin by targeting Glut1. 

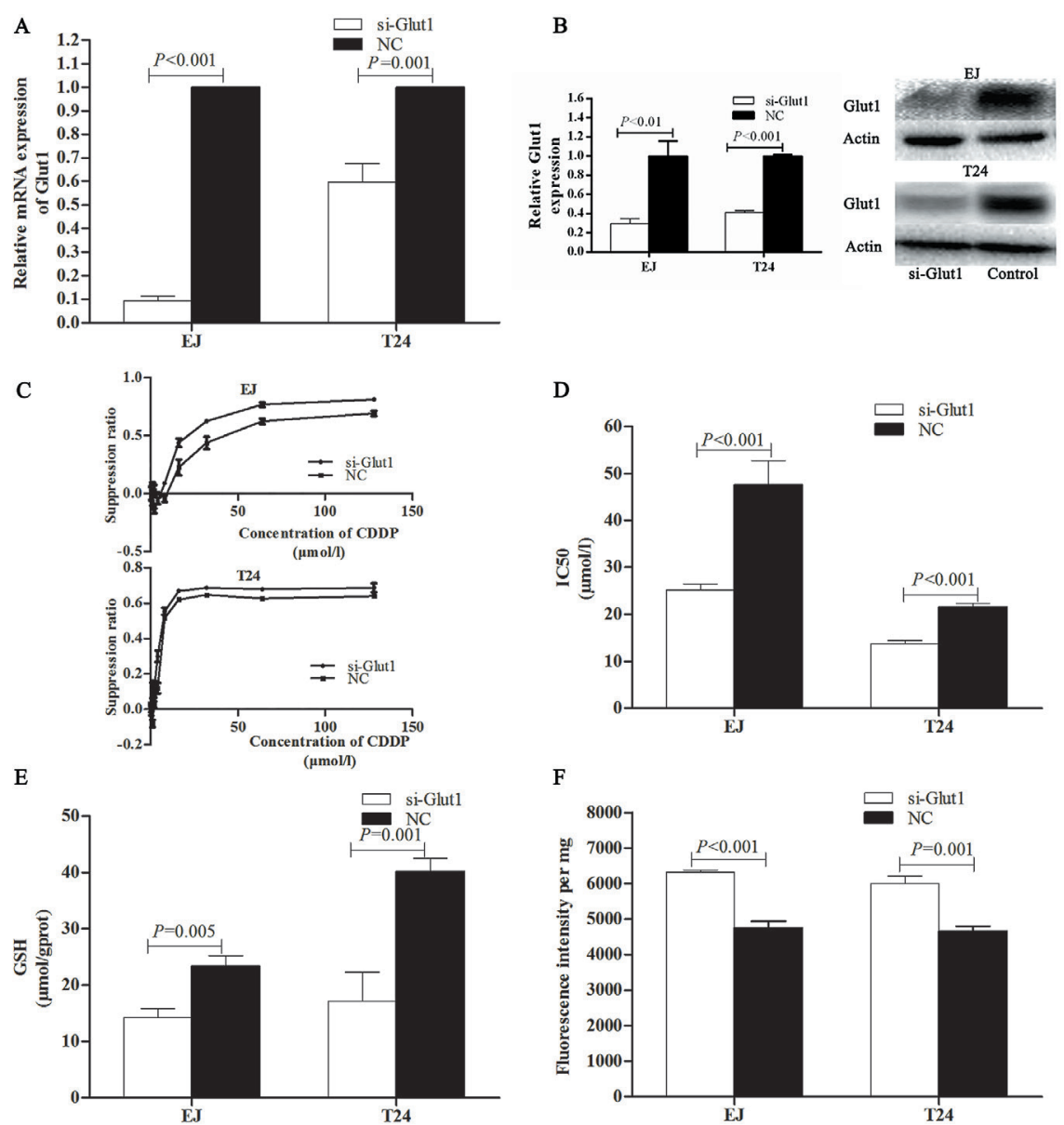

Fig. 3. Knockdown of Glut1 increases the sensitivity to cisplatin of T24 and EJ cells. (A\&B) mRNA and protein levels Glut1 in si-Glut1-transfected and miR-control-transfected cells. (C) Cell viability was determined by the CCK-8 method to be inhibited in si-Glut1-transfected cells in a series of dilute concentrations of cisplatin. (D) Knockdown of Glut1 increased the sensitivity of cells to cisplatin. (E\&F) Increased levels of ROS and reduced GSH were observed in si-Glut1-transfected cells.

Over-expression of Glut1 rescues chemoresistance in miR-218 transfected EJ and T24 cells

To further confirm that miR-218 regulates cisplatin chemo-sensitivity in bladder cancer by targeting Glut1, we performed a restoration experiment through transfecting Glut1 and miR-218 into bladder cancer cells at the same time. IC50 determination assay showed that upregulation of Glut1 restored the chemo-sensitivity induced by overexpressing of miR-218 in EJ and T24 cells (Fig. 5A). In addition, the decreased generation of intracellular GSH and increased level of ROS due to miR-218 were reversed by transfecting Glut1 into EJ and T24 cells (Fig. 5B, 5C). Moreover, transfection of Glut1 into cells rescued the miR-218-induced downregulation of Glut1 at different concentrations of miR-218 (Fig. 5D). In summary, these results indicated that miR-218 play a crucial role in regulating the sensitivity to cisplatin in bladder cancer cells by directly targeting Glut1.

\section{Discussion}

Herein, we demonstrate a novel regulatory signalling mechanism, the miR-218- Glut1 axis, which plays a crucial role in linking miRNAs with cisplatin sensitivity. Our results 

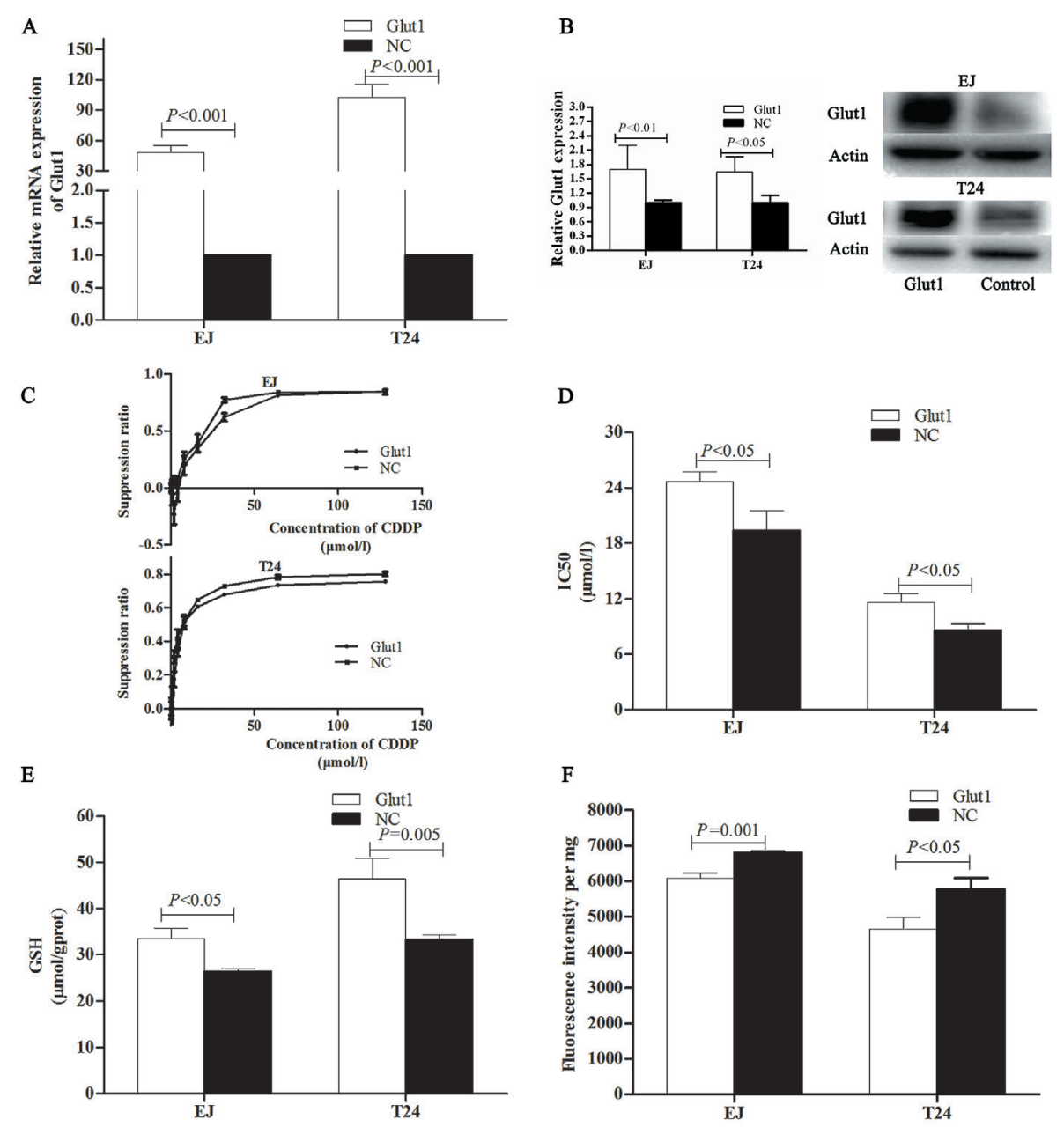

Fig. 4. Over-expressed Glut1 enhances drug-resistance in T24 and EJ cells. (A\&B) mRNA and protein levels of Glut1 in Glut1-transfected and miR-control-transfected cells. (C) Cell viability was determined by the CCK-8 method to be enhanced in Glut1-transfected cells in a series of dilute concentrations of cisplatin. (D) Overexpression of Glut1 increased the resistance of cells to cisplatin. (E\&F) Increased levels of GSH and reduced ROS were observed in Glut1-transfected cells.

indicated that up-regulated miR-218 could increase the cisplatin sensitivity of bladder cancer cells accompanied by reduced glucose uptake, total cellular GSH content and enhanced ROS in T24 and EJ cells. These findings demonstrate that dysregulation of miR-218 could contribute to the decreased sensitivity of bladder cancer cells to cisplatin by glucose metabolism.

Chemotherapy agents, especially cisplatin, are widely used in the management of bladder cancer to reduce relapse, prevent progress and improve survival. However, many tumours have developed chemo-resistance towards cisplatin, a problem that has become a major obstacle in managing bladder cancer. Explorations of the relationship between miRNAs and tumour chemo-sensitivity, in particular, have recently become a research hotspot. Previous studies have confirmed that miR-218 serves as a tumour suppressor in numerous human cancers and over-expressed miR-218 could inhibit the growth and regulate chemo-sensitivity in certain tumours, including gastric cancer and cervical cancer $[25,26]$. It is well known that one miRNA may target many genes in different cell systems and miR-218 is no exception. There are several relatively common target genes of miR-218 in cancers such as BMI-1 [14, 27, 28], surviving [29-32] and HMGB1 [26, 33-36]. BMI-1 is a member of the polycomb group (PcG) of genes and is the first identified PcG transcriptional 


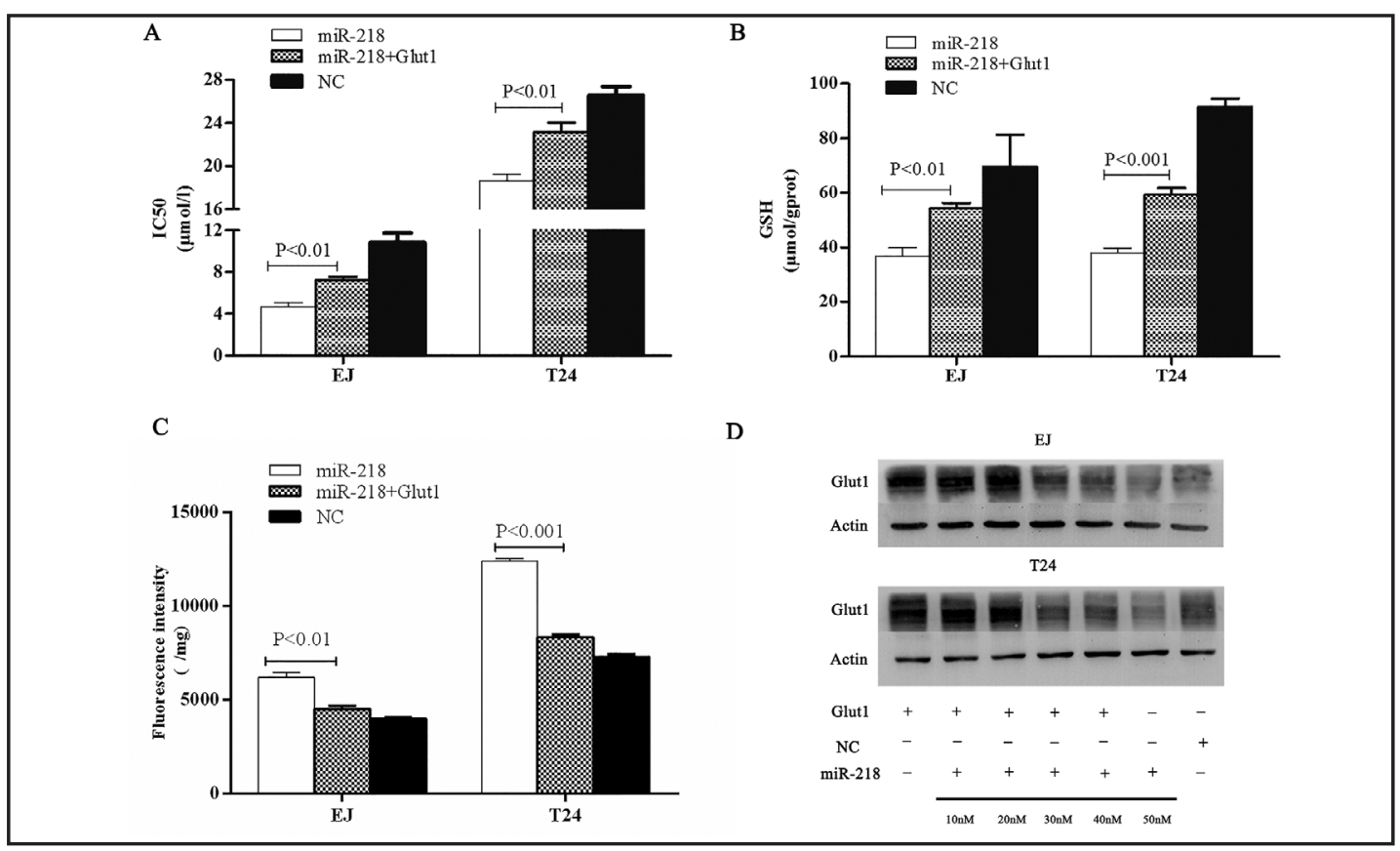

Fig. 5. Over-expression of Glut1 rescues chemoresistance in miR-218 transfected EJ and T24 cells. (A) Upregulation of Glut1 restored the chemo-sensitivity induced by overexpressing of miR-218 in EJ and T24 cells. (B\&C) The reduced GSH and increased level of ROS due to miR-218 were reversed by transfecting Glut1 into EJ and T24 cells. (D) Overexpression of Glut1 rescued the miR-218-induced downregulation of Glut1at different concentrations of miR-218.

repressor. Survivin, the smallest member of the inhibitor of apoptosis (IAP) family, severs as an oncogene due to its anti-apoptotic properties. HMGB1 belongs to the high mobility group protein superfamily and play important roles in the malignant behaviors in cancers. MiR218 functions as a tumour suppressor by targeting BMI-1, survivin or HMGB1 in different types of cancers. Moreover, miR-218 has been found to regulate chemosensitivity in lung cancer[30] , pancreatic cancer[26] and overexpression of miR-218 could induce apoptosis and increase sensitivity to chemotherapeutics in breast cancer by targeting surviving [32]. In our previous study, we found that miR-218 was significantly down-regulated in bladder cancer tissues and that miR-218 could inhibit bladder cancer cell proliferation, migration and invasion by targeting BMI-1 [14]. In the present study, we investigated the association between miR-218 and the sensitivity of T24 and EJ cells to cisplatin by transfecting miR-218 mimics into T24 and EJ cells.

Our results showed that over-expression of miR-218 could dramatically inhibit the proliferation of T24 and EJ cells and promote their sensitivity to cisplatin. Reduced generation of GSH and increased levels of ROS were also observed in cells transfected with miR-218 mimics. GSH generation depends on the rate of glucose uptake, and Glut1 is a key rate-limiting factor in the transport of glucose into cancer cells, playing important roles in tumourigenesis and tumour progress [16]. Glut1 is over-expressed in a variety of malignancies including bladder cancer, [24, 37-40] and over-expression of Glut1 enhances glycolytic activity, increases cancer cell proliferation and promotes the invasion and metastatic proficiency of cancers $[20,21]$. Similarly, our previous study showed that Glut1 was up-regulated in bladder cancer tissues compared with that in adjacent normal tissues [24]. As such, we explored the function of Glut1 and its relationship with the GSH-ROS balance in bladder cancer cells.

A number of studies have recently found that glucose restriction during chemotherapy potentiates sensitivity to chemotherapy agents in breast cancer, melanoma and neuroblastoma in vivo [41]. In addition, research has demonstrated that Glut1 may confer 


\section{Cellular Physiology Cell Physiol Biochem 2017;41:921-932

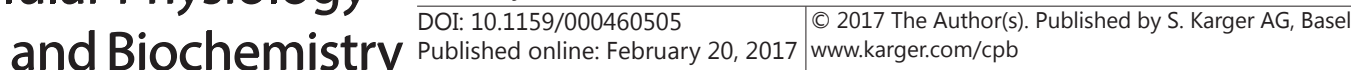 \\ Li et al.: Sensitization of Bladder Cancer to Cisplatin by miR-218-Glut1 Pathway}

resistance to chemotherapy agents in a hypoxia-independent manner [42]. Consistent with previous studies, our results showed that over-expression of Glut1 could promote the proliferation of T24 and EJ cells in a series of dilute concentrations of cisplatin and enhance their chemo-resistance to cisplatin. We also observed dramatically increased levels of GSH and reduced ROS in cells over-expressing Glut1. Intracellular GSH is a major endogenous antioxidant that protects cells from oxidative injury. GSH is considered to play a vital role in regulating the chemo-sensitivity of tumour cells [43-48]. Intracellular levels of ROS have also been found to be closely related to the chemo-sensitivity of cancer cells $[49,50]$. Our results verify these conclusions to a deeper extent. In contrast, siRNA-induced silence of Glut1 generates opposite effects to those over-expressing Glut1 in T24 and EJ cells. Assays showed more sensitivity to cisplatin, reduced GSH and increased ROS production in T24 and EJ cells transfected with si-Glut1.

Bioinformatics analysis has demonstrated that Glut1 is a potential direct target of miR218 in bladder cancer. In the present study, dual luciferase reporter assay confirmed that miR-218 directly targets Glut1. qRT-PCR and Western blot experiments further implied that over-expression of miR-218 could prominently down-regulate the expression level of Glut1 in T24 and EJ cells at both the mRNA and protein levels. Moreover, as mentioned above, overexpressed miR-218 shows effects similar to those observed when cells are transfected with si-Glut1. These results indicate that miR-218 could promote sensitivity to cisplatin in T24 and EJ cell lines by targeting Glut1.

Based on our previous study $[14,24]$ and the present findings, we conclude that restoration of reduced miR-218 levels enhances the sensitivity of bladder cancer cells to cisplatin by directly targeting Glut1. Target miR-218 and Glut1 may provide a potential strategy to restore chemo-sensitivity in bladder cancer.

\section{Acknowledgements}

This work was supported by the National Natural Science Foundation of China (grants No. 81272832 ,81201997 and 81602235), the Priority Academic Program Development of Jiangsu Higher Education Institutions (PAPD) and Jiangsu Provincial Special Program of Medical Science (BL2012027). The funders had no role in study design, data collection and analysis, decision to publish, or preparation of the manuscript.

\section{Disclosure Statement}

Neither author has a competing interest to disclose.

\section{References}

$\checkmark 1$ Ploeg M, Aben KK, Kiemeney LA: The present and future burden of urinary bladder cancer in the world. World J Urol 2009;27:289-293.

- Amling CL: Diagnosis and management of superficial bladder cancer. Curr Probl Cancer 2001;25:219-278.

-3 Witjes JA, Comperat E, Cowan NC, De Santis M, Gakis G, Lebret T, Ribal MJ, Van der Heijden AG, Sherif A, European Association of U: EAU guidelines on muscle-invasive and metastatic bladder cancer: summary of the 2013 guidelines. Eur Urol 2014;65:778-792.

4 Tanji N, Ozawa A, Miura N, Yanagihara Y, Sasaki T, Nishida T, Kikugawa T, Ikeda T, Ochi T, Shimamoto K, Aoki K, Yokoyama M: Long-term results of combined chemotherapy with gemcitabine and cisplatin for metastatic urothelial carcinomas. Int J Clin Oncol 2010;15:369-375.

5 Chang SS: Re: Accelerated methotrexate, vinblastine, doxorubicin, and cisplatin is safe, effective, and efficient neoadjuvant treatment for muscle-invasive bladder cancer: results of a multicenter phase II study with molecular correlates of response and toxicity. J Urol 2015;193:1932.

6 Dasari S, Tchounwou PB: Cisplatin in cancer therapy: molecular mechanisms of action. Eur J Pharmacol 2014;740:364-378. 


\section{Cellular Physiology Cell Physiol Biochem 2017;41:921-932

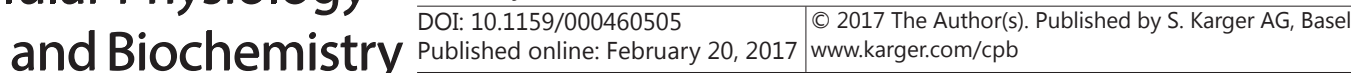

Li et al.: Sensitization of Bladder Cancer to Cisplatin by miR-218-Glut1 Pathway

7 Galluzzi L, Senovilla L, Vitale I, Michels J, Martins I, Kepp 0, Castedo M, Kroemer G: Molecular mechanisms of cisplatin resistance. Oncogene 2012;31:1869-1883.

8 Ma J, Dong C, Ji C: MicroRNA and drug resistance. Cancer Gene Ther 2010;17:523-531.

-9 Zhi Y, Pan J, Shen W, He P, Zheng J, Zhou X, Lu G, Chen Z, Zhou Z: Ginkgolide B Inhibits Human Bladder Cancer Cell Migration and Invasion Through MicroRNA-223-3p. Cell Physiol Biochem 2016;39:1787-1794.

10 Sun DK, Wang JM, Zhang P, Wang YQ: MicroRNA-138 Regulates Metastatic Potential of Bladder Cancer Through ZEB2. Cell Physiol Biochem 2015;37:2366-2374.

11 Gu JJ, Gao GZ, Zhang SM: miR-218 inhibits the migration and invasion of glioma U87 cells through the Slit2Robo1 pathway. Oncol Lett 2015;9:1561-1566.

12 Li J, Ping Z, Ning H: MiR-218 impairs tumor growth and increases chemo-sensitivity to cisplatin in cervical cancer. Int J Mol Sci 2012;13:16053-16064.

13 Zhang X, Shi H, Tang H, Fang Z, Wang J, Cui S: miR-218 inhibits the invasion and migration of colon cancer cells by targeting the PI3K/Akt/mTOR signaling pathway. Int J Mol Med 2015;35:1301-1308.

14 Cheng Y, Yang X, Deng X, Zhang X, Li P, Tao J, Lu Q: MicroRNA-218 inhibits bladder cancer cell proliferation, migration, and invasion by targeting BMI-1. Tumour Biol 2015;36:8015-8023.

15 Moreno-Sanchez R, Rodriguez-Enriquez S, Marin-Hernandez A, Saavedra E: Energy metabolism in tumor cells. FEBS J 2007;274:1393-1418.

16 Koch A, Lang SA, Wild PJ, Gantner S, Mahli A, Spanier G, Berneburg M, Muller M, Bosserhoff AK, Hellerbrand C: Glucose transporter isoform 1 expression enhances metastasis of malignant melanoma cells. Oncotarget 2015;6:32748-32760.

17 Amann T, Maegdefrau U, Hartmann A, Agaimy A, Marienhagen J, Weiss TS, Stoeltzing O, Warnecke C, Scholmerich J, Oefner PJ, Kreutz M, Bosserhoff AK, Hellerbrand C: GLUT1 expression is increased in hepatocellular carcinoma and promotes tumorigenesis. Am J Pathol 2009;174:1544-1552.

-18 Shen YM, Arbman G, Olsson B, Sun XF: Overexpression of GLUT1 in colorectal cancer is independently associated with poor prognosis. Int J Biol Markers 2011;26:166-172.

19 Younes M, Juarez D, Lechago LV, Lerner SP: Glut 1 expression in transitional cell carcinoma of the urinary bladder is associated with poor patient survival. Anticancer Res 2001;21:575-578.

20 Liu Y, Zhang W, Cao Y, Liu Y, Bergmeier S, Chen X: Small compound inhibitors of basal glucose transport inhibit cell proliferation and induce apoptosis in cancer cells via glucose-deprivation-like mechanisms. Cancer Lett 2010;298:176-185.

-21 Zhang W, Liu Y, Chen X, Bergmeier SC: Novel inhibitors of basal glucose transport as potential anticancer agents. Bioorg Med Chem Lett 2010;20:2191-2194.

22 Tao J, Lu Q, Wu D, Li P, Xu B, Qing W, Wang M, Zhang Z, Zhang W: microRNA-21 modulates cell proliferation and sensitivity to doxorubicin in bladder cancer cells. Oncol Rep 2011;25:1721-1729.

-23 Ishimoto T, Nagano O, Yae T, Tamada M, Motohara T, Oshima H, Oshima M, Ikeda T, Asaba R, Yagi H, Masuko T, Shimizu T, Ishikawa T, Kai K, Takahashi E, Imamura Y, Baba Y, Ohmura M, Suematsu M, Baba H, Saya $\mathrm{H}$ : CD44 variant regulates redox status in cancer cells by stabilizing the xCT subunit of system xc(-) and thereby promotes tumor growth. Cancer Cell 2011;19:387-400.

-24 Yang X, Cheng Y, Li P, Tao J, Deng X, Zhang X, Gu M, Lu Q, Yin C: A lentiviral sponge for miRNA-21 diminishes aerobic glycolysis in bladder cancer T24 cells via the PTEN/PI3K/AKT/mTOR axis. Tumour Biol 2015;36:383-391.

-25 Fan R, Zhong J, Zheng S, Wang Z, Xu Y, Li S, Zhou J, Yuan F: microRNA-218 increase the sensitivity of gastrointestinal stromal tumor to imatinib through PI3K/AKT pathway. Clin Exp Med 2015;15:137-144.

-26 Liu Z, Du R, Long J, Guo K, Ge C, Bi S, Xu Y: microRNA-218 promotes gemcitabine sensitivity in human pancreatic cancer cells by regulating HMGB1 expression. Chin J Cancer Res 2015;27:267-278.

27 Fu WM, Tang LP, Zhu X, Lu YF, Zhang YL, Lee WY, Wang H, Yu Y, Liang WC, Ko CH, Xu HX, Kung HF, Zhang JF: MiR-218-targeting-Bmi-1 mediates the suppressive effect of 1,6,7-trihydroxyxanthone on liver cancer cells. Apoptosis 2015;20:75-82.

28 He X, Dong Y, Wu CW, Zhao Z, Ng SS, Chan FK, Sung JJ, Yu J: MicroRNA-218 inhibits cell cycle progression and promotes apoptosis in colon cancer by downregulating BMI1 polycomb ring finger oncogene. Mol Med 2013;18:1491-1498.

29 Kogo R, How C, Chaudary N, Bruce J, Shi W, Hill RP, Zahedi P, Yip KW, Liu FF: The microRNA-218 Survivin axis regulates migration, invasion, and lymph node metastasis in cervical cancer. Oncotarget 2015;6:10901100. 


\section{Cellular Physiology Cell Physiol Biochem 2017:41:921-932 \begin{tabular}{ll|l} 
and Biochemistry 10.1159/000460505 & $\begin{array}{l}\text { @ } 2017 \text { The Author(s). Published by S. Karger AG, Basel } \\
\text { www.karger.com/cpb }\end{array}$ \\
\hline
\end{tabular}

30 Zarogoulidis P, Petanidis S, Kioseoglou E, Domvri K, Anestakis D, Zarogoulidis K: MiR-205 and miR-218 expression is associated with carboplatin chemoresistance and regulation of apoptosis via Mcl-1 and Survivin in lung cancer cells. Cell Signal 2015;27:1576-1588.

-31 Alajez NM, Lenarduzzi M, Ito E, Hui AB, Shi W, Bruce J, Yue S, Huang SH, Xu W, Waldron J, O'Sullivan B, Liu FF: MiR-218 suppresses nasopharyngeal cancer progression through downregulation of survivin and the SLIT2-ROBO1 pathway. Cancer Res 2011;71:2381-2391.

-32 Hu Y, Xu K, Yague E: miR-218 targets survivin and regulates resistance to chemotherapeutics in breast cancer. Breast Cancer Res Treat 2015;151:269-280.

-33 Gu J, Xu R, Li Y, Zhang J, Wang S: MicroRNA-218 modulates activities of glioma cells by targeting HMGB1. Am J Transl Res 2016;8:3780-3790.

34 Liu Z, Xu Y, Long J, Guo K, Ge C, Du R: microRNA-218 suppresses the proliferation, invasion and promotes apoptosis of pancreatic cancer cells by targeting HMGB1. Chin J Cancer Res 2015;27:247-257.

-35 Ran X, Yang J, Liu C, Zhou P, Xiao L, Zhang K: MiR-218 inhibits HMGB1-mediated autophagy in endometrial carcinoma cells during chemotherapy. Int J Clin Exp Pathol 2015;8:6617-6626.

-36 Zhang C, Ge S, Hu C, Yang N, Zhang J: MiRNA-218, a new regulator of HMGB1, suppresses cell migration and invasion in non-small cell lung cancer. Acta Biochim Biophys Sin (Shanghai) 2013;45:1055-1061.

37 Palit V, Phillips RM, Puri R, Shah T, Bibby MC: Expression of HIF-1alpha and Glut-1 in human bladder cancer. Oncol Rep 2005;14:909-913.

-38 Sakashita M, Aoyama N, Minami R, Maekawa S, Kuroda K, Shirasaka D, Ichihara T, Kuroda Y, Maeda S, Kasuga M: Glut1 expression in T1 and T2 stage colorectal carcinomas: its relationship to clinicopathological features. Eur J Cancer 2001;37:204-209.

-39 Medina RA, Owen GI: Glucose transporters: expression, regulation and cancer. Biol Res 2002;35:9-26.

40 Chang S, Lee S, Lee C, Kim JI, Kim Y: Expression of the human erythrocyte glucose transporter in transitional cell carcinoma of the bladder. Urology 2000;55:448-452.

-41 Lee C, Raffaghello L, Brandhorst S, Safdie FM, Bianchi G, Martin-Montalvo A, Pistoia V, Wei M, Hwang S, Merlino A, Emionite L, de Cabo R, Longo VD: Fasting cycles retard growth of tumors and sensitize a range of cancer cell types to chemotherapy. Sci Transl Med 2012;4:124ra127.

42 Airley RE, Phillips RM, Evans AE, Double J, Burger AM, Feibig HH, West CM, Stratford IJ: Hypoxia-regulated glucose transporter Glut-1 may influence chemosensitivity to some alkylating agents: results of EORTC (First Translational Award) study of the relevance of tumour hypoxia to the outcome of chemotherapy in human tumour-derived xenografts. Int J Oncol 2005;26:1477-1484.

43 Tai DJ, Jin WS, Wu CS, Si HW, Cao XD, Guo AJ, Chang JC: Changes in intracellular redox status influence multidrug resistance in gastric adenocarcinoma cells. Exp Ther Med 2012;4:291-296.

44 Shi J, Sun B, Shi W, Zuo H, Cui D, Ni L, Chen J: Decreasing GSH and increasing ROS in chemosensitivity gliomas with IDH1 mutation. Tumour Biol 2015;36:655-662.

-45 Juvekar AS, Adwankar MK, Tongaonkar HB: Effect of cisplatin-based chemotherapy on emergence of cisplatin resistance, and its correlation with intracellular glutathione levels and accumulation of p53 protein in human ovarian cancer. Cancer Biother Radiopharm 2000;15:295-300.

-46 Izaki S, Goto H, Yokota S: Increased chemosensitivity and elevated reactive oxygen species are mediated by glutathione reduction in glutamine deprived neuroblastoma cells. J Cancer Res Clin Oncol 2008;134:761768.

47 Hamberger J, Liebeke M, Kaiser M, Bracht K, Olszewski U, Zeillinger R, Hamilton G, Braun D, Bednarski PJ: Characterization of chemosensitivity and resistance of human cancer cell lines to platinum(II) versus platinum(IV) anticancer agents. Anticancer Drugs 2009;20:559-572.

48 Yamagata K, Kondo Y: [A study on the relationship between sensitivity to CDDP and inducibility of metallothionein and glutathione of genitourinary tumors]. Nihon Hinyokika Gakkai Zasshi 1995;86:15751582.

49 Ma J, Yang J, Wang C, Zhang N, Dong Y, Wang C, Wang Y, Lin X: Emodin augments cisplatin cytotoxicity in platinum-resistant ovarian cancer cells via ROS-dependent MRP1 downregulation. Biomed Res Int 2014;2014:107671.

50 Huang XZ, Wang J, Huang C, Chen YY, Shi GY, Hu QS, Yi J: Emodin enhances cytotoxicity of chemotherapeutic drugs in prostate cancer cells: the mechanisms involve ROS-mediated suppression of multidrug resistance and hypoxia inducible factor-1. Cancer Biol Ther 2008;7:468-475. 